\title{
Evidence of Encoding Specificity for the Effects of Synopses on the Recognition of Believable and Unbelievable News Headlines
}

\author{
Gibbons JA1*, Lukowski AF², Walsh $\mathrm{KM}^{3}{ }^{3}$, Smeland $\mathrm{JN}^{4}$ and Smith \\ $\mathrm{CL}^{5}$ \\ 1Department of Psychology, Christopher Newport University, USA \\ 2Department of Psychology and Social Behavior, University of California-Irvine, USA \\ ${ }^{3}$ College of Arts and Sciences, Emory University, USA \\ ${ }^{4}$ Department of Psychology, Christopher Newport University, USA \\ ${ }^{5}$ Department of Psychology, Christopher Newport University, USA
}

\section{Research Article \\ Volume 3 Issue 7}

Received Date: August 06, 2018

Published Date: August 14, 2018

*Corresponding author: Jeffrey A Gibbons, Department of Psychology, Christopher Newport University , Avenue of the Arts, Newport News, VA 23606, Tel: (757) 594-7256; Email: jgibbons@cnu.edu

\begin{abstract}
Gibbons, Lukowski, and Walker (2005) argued that elaborate processing explained the finding that unbelievable news headlines were recognized better than believable news headlines, and they suggested that headline synopses may further enhance processing. In Experiment 1, we tested whether elaborative synopses at presentation (i.e., learning) enhanced recognition, and we found that they decreased recognition. Instead of elaborate processing, these results were congruent with encoding specificity. In Experiments 2-5, we used reiterative synopses to create matching and mismatching synopsis conditions at presentation and test to assess encoding specificity. The results for Experiments 2-4 demonstrated encoding specificity for believable headlines, and the results for Experiment 5 showed encoding specificity for believable and unbelievable headlines. These results provide some support for encoding specificity in the realm of media headlines.
\end{abstract}

Keywords: News headlines; Synopses; Recognition; Headline believability

\section{Introduction}

The Information Age provides the consumer with attention-grabbing information via television, radio, and the Internet [1,2]. Grabe and colleagues (2000) suggested that unbelievable information on television and in printed media tends to focus consumers' attention, which may further enhance processing. In an effort to understand the underlying mechanisms responsible for processing news headlines, Gibbons, Lukowski, and Walker (2005) examined the effect of believable and unbelievable news headlines on recognition and they found that participants recognized unbelievable headlines more slowly but more accurately than believable headlines. Gibbons et al. concluded that unbelievable headlines grabbed consumers' attention and enhanced recognition via elaborate processing, as suggested by the levels of processing (LOP) model [3-6]. Gibbons, et al. suggested that coupling synopses with headlines at learning might further enhance cognitive processing. To evaluate the explanatory power of the LOP model, we manipulated the presence of synopses at presentation to examine their 


\section{Psychology \& Psychological Research International Journal}

effect on the recognition of believable and unbelievable news headlines.

A few researchers have considered the cognitive mechanisms related to the processing of believable and unbelievable print media [1,2,7]. For example, Grabe et al. (2000) gave participants eight entire news stories that were either written in a tabloid (typically unbelievable) format or in the format of a traditional news story (typically believable). Recognition was assessed shortly after stimulus presentation; recall was assessed by phone 2 days later. Grabe, et al. found that the tabloid or unbelievable stories increased attention and arousal at encoding, but this increased attention did not significantly aid the recognition or recall of these stories. Although one would expect increased recognition and recall with increased attention, the increased arousal may have acted as a distraction or attention may not have increased to a sufficient level to increase recognition and recall.

Extending the work of Grabe, et al. (2000), Gibbons et al. (2005) evaluated the power of the limited capacity model of mediated message processing to explain the effect of exposure on the believability and recognition of believable and unbelievable news headlines. The limited capacity of mediated message processing suggests that arousing stimuli increase attention and long-term memory via automatic processing $[7,8]$. Gibbons et al. (2005) found that participants took longer to make believability ratings and recognition judgments for unbelievable headlines than for believable headlines and they recognized unbelievable headlines more accurately than believable headlines [1]. In addition, prolonged exposure enhanced the believability of unbelievable headlines. Instead of supporting the limited capacity model of mediated message processing, the results suggested that the unbelievable headlines contained unique and salient characteristics that allowed for deep or elaborate (i.e., effortful) processing (as suggested by the LOP framework).

Gibbons and colleagues (2005) did suggest a further experiment to test their elaborate processing explanation for the effects of headline believability on cognitive processing. Specifically, Gibbons and colleagues suggested that researchers could add synopses to believable and unbelievable headlines at presentation and examine recognition and believability judgments across presentation (i.e., learning/encoding) and test. Based on the LOP framework, the researchers predicted that the synopses should increase the processing and, hence, the recognition of believable and unbelievable headlines.

\section{Experiment 1}

Experiment 1 was designed to test the effect of headline synopses at presentation on the recognition of believable and unbelievable news headlines. To facilitate the degree that synopses could enhance elaborative processes at presentation (i.e., encoding/learning) and increase recognition (i.e., as suggested by Gibbons et al., 2005), we used elaborative synopses at presentation. Elaborative synopses were comprised of news stories that provided details beyond the ones presented in a headline. Elaborative synopses were added to half of the believable and unbelievable news headlines. Although recognition was the focus on the current study, participants rated the believability of unbelievable and believable headlines at learning and at test. The act of making believability ratings at learning helped ensure that the participants attended to and processed the headlines [1].

At test, participants rated the believability of the previously presented headlines as well as foil (new) headlines. After participants provided a believability rating for a headline, they provided a recognition judgment for that headline by circling it if they recognized it from presentation. No headlines were accompanied by synopses at test. According to the elaborate processing explanation, elaborative synopses were expected to increase recognition, perhaps by deepening and enhancing cognitive processes.

\section{Method}

\section{Participants}

The participants consisted of 41 undergraduate psychology students enrolled in classes at a small, public southeastern university seeking extra credit. All participants were treated in accordance with the "Ethical principles of psychologist and code of conduct" [9].

\section{Materials and Procedure}

At encoding, participants received a questionnaire containing 20 headlines (10 initially believable and 10 initially unbelievable headlines) as well as 10 relevant article synopses (Appendix A): Five synopses followed initially believable headlines and five synopses followed initially unbelievable headlines. Initially believable headlines were selected verbatim from newspapers and initially unbelievable headlines were selected verbatim from tabloids. The wording used for the synopses of each type of headline were generally selected from the article in which the headline was found, but the synopses were limited to the most important points made in the article to 


\section{Psychology \& Psychological Research International Journal}

control the length of the synopses. The headlines and synopses were collected from a variety of newspapers and tabloids found in hard copy and via the internet.

Participants read each headline and rated its believability on a scale ranging from -3 (extremely unbelievable) to +3 (extremely believable). Once each participant had finished rating the believability of the headlines, the researcher collected the questionnaires. Two days later at test, the same participants completed a 40-item questionnaire (Appendix B), which contained 20 initially believable headlines (10 headlines that were presented at presentation and 10 new headlines) and 20 initially unbelievable headlines (10 headlines that were presented at presentation and 10 new headlines). Article synopses were not present at test. Participants once again rated the believability of each headline (using the same scale as before) as well as circled the headlines that they recognized from presentation. Once this process was complete, the researchers collected the surveys and the participants were debriefed.

\section{Measures, Design, and Analyses}

Although believability ratings were obtained, recognition was the focus of the current study. Hits and A' (hits corrected for false alarms) operated as the primary dependent variables. The groups pertained to the four headline types: believable headlines with synopses at presentation, believable headlines without synopses at presentation, unbelievable headlines with synopses at presentation, and unbelievable headlines without synopses at presentation. The proportion of hits was calculated for the four headline types at test by dividing the number of circled/recognized targets by 5 , which was the total number of items within each headline group.

The proportion of false alarms was calculated for believable and unbelievable headlines from the foils at test by dividing the number of circled (incorrectly recognized) foils for a headline type by the total number of foils (10 for each type of headline). $A^{\prime}$ was then calculated from the hits and false alarms based on Snodgrass and Corwin (1988; $\mathrm{A}^{\prime}=.5+[(\mathrm{H}-\mathrm{FA})(1+\mathrm{H}-$ $\mathrm{FA})] /[(4 \mathrm{H}(1-\mathrm{FA})])$. The false alarm rates were low with a rate of .012 overall, and low rates of .010 and .015 for initially believable and initially unbelievable headlines, respectively. The proportion of hits and the $\mathrm{A}^{\prime}$ measure of recognition were placed into a 2 (Headline Type) x 2 (Synopsis) repeated-measures design and the data were analyzed via ANOVA, and follow-up $t$-tests [10].

\section{Results}

The general alpha level was set at .05. As this research is the first to examine the recognition of news headlines and synopses in this fashion, we further analyzed predicted interactions with follow-up $t$-tests.

\section{Hits}

Initially unbelievable headlines $(M=0.849, S E=0.023)$ were recognized significantly more accurately than initially believable headlines $(M=0.739, S E=0.032), F(1$, $40)=26.129, p<.001$, power $=.999, \eta^{2}=.395$. Moreover, the headlines followed by synopses at presentation $(M=$ $0.759, S E=0.034$ ) were recognized less accurately than the headlines not followed by synopses at presentation $(M=0.829, S E=0.023), F(1,40)=7.044, p=.011$, power $=$ $.736, \eta^{2}=.150$.

\section{$\mathbf{A}^{\prime}$}

$A^{\prime}$ cannot be calculated if the hit and false alarm rates are both 0 for a particular condition. Although there were no cases of 0 values for any participants, the complete data set across all the conditions could not be calculated for 1 participant (i.e., data were missing), so the data for this individual were excluded from the analyses. Initially unbelievable headlines $(M=.959, S E=.006)$ were recognized significantly better than initially believable headlines $(M=.933, S E=.008), F(1,39)=24.782, p<.001$, power $=.998, \eta^{2}=.389$. Moreover, the headlines followed by synopses at presentation $(M=.938, S E=.008)$ were recognized less accurately than the headlines not followed by synopses at presentation $(M=.954, S E=$ .006), $F(1,39)=6.028, p=.019$, power $=.668, \eta^{2}=.134$.

\section{Discussion}

As expected, the results for the hit and $A^{\prime}$ measures showed that unbelievable headlines were recognized better than believable headlines, presumably because unbelievable headlines grabbed participants' attention, which enhanced encoding and memory [11]. Although Grabe, et al. (2000) did not find such increases in recall and recognition for tabloids or unbelievable news stories, even though they demonstrated increased arousal and attention at encoding for these stimuli, these researchers focused on stories as the stimuli rather than headlines. The arousing nature of unbelievable news stories, such as tabloids, may not increase memory for story details because the details are distracting and much more difficult to remember than more general information (e.g., as demonstrated by Thompson, Gibbons, Vogl, \& Walker, 1996), like the information presented in news headlines [12]. 


\section{Psychology \& Psychological Research International Journal}

More importantly, news headlines with synopses were recognized less well than headlines without synopses. As the synopses were present at learning, but not at test, this mismatching of synopses conditions could have produced the low recognition. Rather than supporting elaborative processing models, the results for synopses seem to support encoding specificity, such that matching conditions at presentation and test (i.e., absence of headline synopses) enhanced cognitive processing, whereas mismatching conditions across presentation and test inhibited cognitive processing [13-15]. The literature on encoding specificity generally suggests that congruent conditions across presentation and test produce the best retrieval, and the literature that generalizes the principle to different stimuli and conditions is fairly extensive [1619]. In support of Tulving and Thomson's (1973) claim that the encoding specificity principle pertains to "all known phenomena of episodic memory and retrieval" (p. 370) [15], encoding specificity occurs across recall and recognition [20-24].

In contrast to the large number of articles examining the encoding specificity principle with traditional stimuli, a few researchers have extended the phenomenon to media stimuli, such as television advertisements and news stories $[25,26]$. However, past research has not examined whether encoding specificity can explain the effects of synopses on the recognition of believable and unbelievable news headlines. As an alternative to encoding specificity explaining the poor recognition produced by the presence of synopses only at learning in Experiment 1, the additional information in the elaborative synopses may have distracted participants from encoding the actual headlines, which reduced recognition. Experiment 2 was created to correct this flaw in the synopses and evaluate their effects on recognition when they are present and absent at learning and test.

\section{Experiment 2}

Experiment 2 was designed to replicate and extend/correct the procedure used in Experiment 1 . Specifically, Experiment 2 manipulated the presence of reiterative synopses at presentation and test to fully examine encoding specificity for believable and unbelievable news headlines. Reiterative synopses provided supportive information that complemented the main points made in the headlines (i.e., reworded headline). We used ratings from another sample to ensure that the believability of the believable headlines was similar across the four conditions at test. The same controls were ensured for the unbelievable headlines. If reiterative synopses demonstrate elaborative processing as part of the LOP framework, reiterative synopses at presentation should enhance recognition. If reiterative synopses demonstrate encoding specificity, recognition should be higher for matching synopsis conditions (across presentation and test) than for mismatching synopsis conditions. Based on past research, we expected the results to support both elaborative processing models and encoding specificity.

\section{Method}

\section{Participants}

All 36 students were undergraduate psychology students from a small, public southeastern university who participated in the experiment to earn extra credit. The participants included both male and female adults between 18 and 25 years of age. All participants were treated in accordance to with the "Ethical principles of psychologists and code of conduct" [9].

\section{Materials}

Two main questionnaires were used in the experiment: one for presentation and one for test. The questionnaires contained initially believable headlines and initially unbelievable headlines that listed the headlines in a randomized order that remained constant. Both main questionnaires presented a believability scale for each headline, whereas the test questionnaire also asked participants to circle headlines if they appeared on the presentation questionnaire. The headlines were designated as either initially believable or initially unbelievable based upon the believability ratings obtained from a pilot study. The believability scale ranged from -3 (extremely unbelievable) to +3 (extremely believable), with 0 being neutral. The presentation questionnaire contained 24 items consisting of 12 believable headlines and 12 unbelievable headlines (Appendix C). At presentation, half of the believable headlines and half of the unbelievable headlines were presented with article-relevant synopses. The test questionnaire (Appendix D) contained 48 items consisting of 24 believable headlines ( 12 from the first questionnaire and 12 new headlines) and 24 unbelievable headlines (12 from the first questionnaire and 12 new headlines).

The presentation and test questionnaires were combined to create four synopsis groupings, which included synopses at presentation and test, synopses at presentation but not at test, synopses not at presentation but at test, and synopses neither at presentation nor at test. Specifically, three of the original six believable headlines followed by synopses at presentation were 


\section{Psychology \& Psychological Research International Journal}

again shown with these same synopses at test, whereas three of the original six believable headlines followed by synopses at presentation were not followed by synopses at test. Three of the original six believable headlines not followed by synopses at presentation were shown with synopses at test, whereas three of the original six believable headlines not followed by synopses at presentation were not followed by synopses at test. The unbelievable headlines were paired across presentation and test in the same way that believable headlines were paired.

We created four headline groupings that were similar in believability and placed them in synopsis groupings. The headline groupings were created based on believability ratings from prior studies to ensure that each headline group was as similar in average believability rating as possible across the believable or unbelievable headlines, respectively. Groups of believable and unbelievable headlines were paired to create a headline grouping, such as headline grouping A. We paired a synopsis grouping with a headline grouping, such that synopses appeared at presentation and test for headlines in headline grouping $\mathrm{A}$, synopses appeared at presentation but not test for headlines in headline grouping B, no synopses appeared at presentation but synopses appeared at test for headlines in headline grouping $\mathrm{C}$, and no synopses appeared at presentation nor test for headlines in headline grouping $\mathrm{D}$.

\section{Procedure}

Participants each received a questionnaire containing 24 headlines $(12$ believable headlines and 12 unbelievable headlines) at presentation. Participants read each headline and rated its believability on the scale that followed each headline. Once each participant was finished rating the believability of the headlines, the researcher collected the questionnaires. Two days later at test, the same participants returned and they were each given a 48-item questionnaire, which contained 24 believable headlines and 24 unbelievable headlines. Twelve of the 24 believable headlines were presented at both presentation and test, whereas 12 of the 24 unbelievable headlines were presented at both presentation and test. Participants then rated the believability of each headline as well as circled the headlines that they believed they recognized from the encoding questionnaires.

\section{Measures, Design, and Analyses}

Hits and A' (hits corrected for false alarms) served as the dependent variables in the experiment. Hits were calculated across eight headline categories. These categories included initially believable headlines with synopses present at both presentation and test, initially believable headlines with synopses present only at presentation, initially believable headlines with synopses present only at test, and initially believable headlines with no synopses present at either presentation or test. The headline categories also included initially unbelievable headlines with synopses at both presentation and test, initially unbelievable headlines with synopses present only at presentation, initially unbelievable headlines with synopses present only at test, and initially unbelievable headlines with no synopses present at either presentation or test.

False alarms were calculated across four categories, which included believable and unbelievable headlines with and without synopses at test. $\mathrm{A}^{\prime}$ was computed as described in Experiment 1 . The false alarm rates were low with a rate of .013 overall, and rates of .014, .014, .010, and .014 for believable headlines without synopses, believable headlines with synopses, unbelievable headlines without synopses, and unbelievable headlines with synopses, respectively. The hit and A' measures were placed into a 2 (Headline Type) x 2 (Presence of Synopses at Presentation) x 2 (Presence of Synopses at Test) repeated measures design and the data were analyzed using ANOVA. Follow-up ANOVAs and paired-samples $t$ tests were conducted to understand interactions.

\section{Results}

\section{Hits}

The initially unbelievable headlines $(M=.914, S E=$ .019) were recognized more accurately overall than the initially believable headlines $(M=.799, S E=0.028), F(1$, $35)=26.840, p<.001$, power $=.999, \eta^{2}=.434$. No other main effects were significant. Although none of the 2-way interactions were statistically significant, the Headline Type $x$ Presence of Synopses at Presentation x Presence of Synopses at Test interaction was statistically significant, $F(1,35)=18.846, p<.001$, power $=.988, \eta^{2}=.350$.

A breakdown of the 3-way interaction yielded a significant Presence of Synopses at Presentation $\mathrm{x}$ Presence of Synopses at Test interaction for initially believable headlines, $F(1,35)=16.593, p=.001$, power $=$ $.977, \eta^{2}=.322$, but not for initially unbelievable headlines, $F(1,35)=2.968, p=.094$, power $=.388, \eta^{2}=.078$. Further analysis of the significant 2 -way interaction for initially believable headlines revealed that recognition accuracy was higher for initially believable headlines with congruent conditions when synopses were present at 
presentation $\left(M_{\text {Synopses present at presentation and test }}=.843, S E=\right.$ .039; $M_{\text {Synopses present at presentation but absent at test }}=.741, S E=.042$ ), $t(35)=2.231, p=.032$, power $=.583, \eta^{2}=.124$, and when synopses were absent at presentation (Mynopses absent at presentation but present at test $=.713, S E=.042 ; M_{\text {Synopses absent at }}$ presentation and test $=.898, S E=.037), t(35)=-3.548, p=.001$, power $=.932, \eta^{2}=.265$. The hit results for the initially believable headlines demonstrate full encoding specificity, such that matching synopsis conditions across presentation and test produced the highest recognition accuracy.

\section{$\mathbf{A}^{\prime}$}

A' could not be calculated for 4 participants; the data for these participants were dropped from the overall $A^{\prime}$ analyses. The initially unbelievable headlines $(M=.981$, $S E=.003$ ) were better recognized than the initially believable headlines $(M=.955, S E=.006), F(1,31)=$ $16.137, p<.001$, power $=.973, \eta^{2}=.342$. No other main effects were significant. Of the 2-way interactions, only the Presence of Synopses at Presentation $x$ Presence of Synopses at Test interaction was statistically significant, $F(1,31)=7.066, p=.012$, power $=.731, \eta^{2}=.186$. Moreover, the Headline Type $\mathrm{x}$ Presence of Synopses at Presentation $x$ Presence of Synopses at Test interaction was statistically significant, $F(1,31)=20.349, p<.001$, power $=.992, \eta^{2}=.396$ (see Figures $1 \mathrm{~A}$ and $\left.1 \mathrm{~B}\right)$.

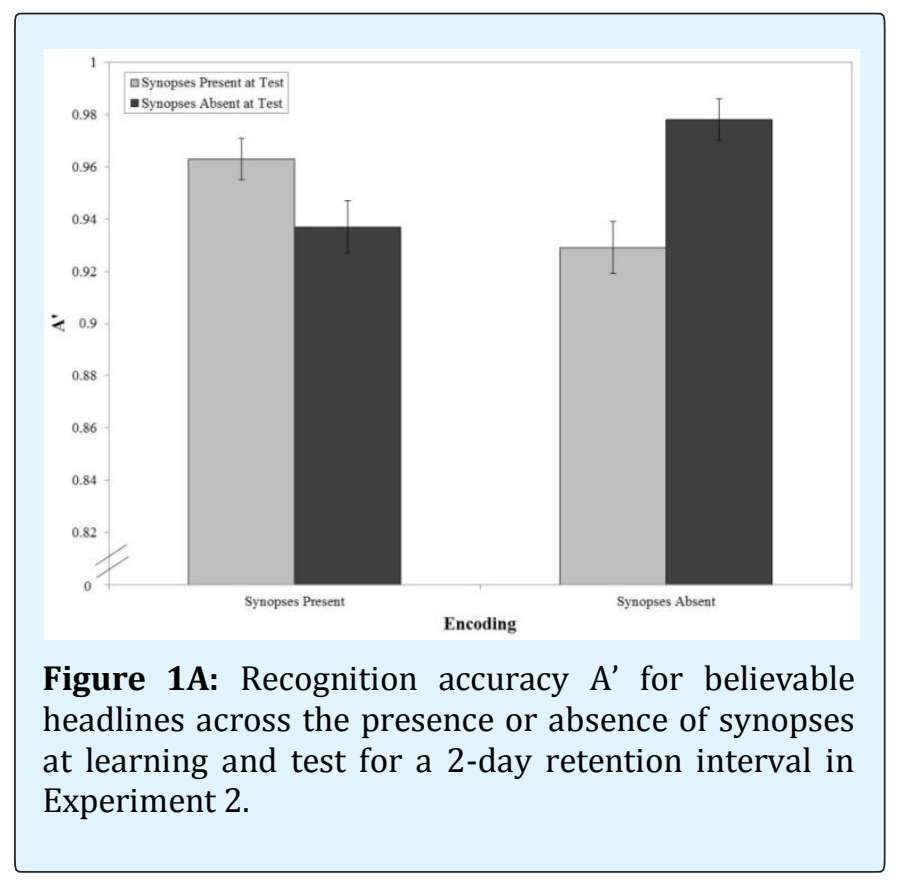

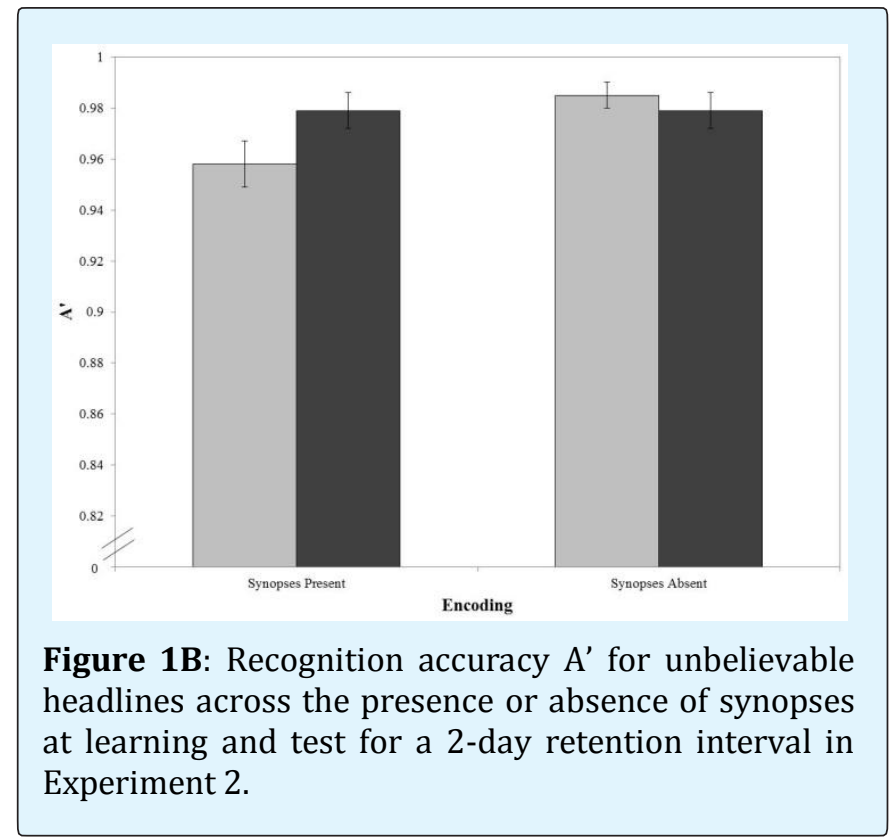

Further analysis of the 3-way interaction yielded a statistically significant Presence of Synopses at Presentation $x$ Presence of Synopses at Test interaction for initially believable headlines, $F(1,31)=26.096, p<$ .001 , power $=.999, \eta^{2}=.457$ (Figure 1A), but not for initially unbelievable headlines, $F(1,35)=3.150, p=.085$, power $=.408, \eta^{2}=.083$ (Figure 1B). Additional analysis of the 2-way interaction for initially believable headlines using paired samples $t$-tests revealed that recognition was significantly higher for initially believable headlines with congruent conditions when synopses were present at presentation ( $M_{\text {Synopses present at presentation and test }}=.966, S E=$ .008; $M_{\text {Synopses present at presentation and absent at test }}=.934, S E=.011$ ), $t(33)=2.734, p=.010$, power $=.756, \eta^{2}=.185$, and when synopses were absent at presentation (Mynopses absent at presentation but present at test $=.929, S E=.010 ; M_{\text {Synopses absent at }}$ presentation and test $=.976, S E=.008), t(33)=-4.330, p<.001$, power $=.987, \eta^{2}=.362$. The $A^{\prime}$ results for the initially believable headlines demonstrate full encoding specificity, such that matching synopsis conditions across presentation and test produced the highest recognition accuracy.

\section{Discussion}

The presence of synopses at learning did not enhance recognition. Therefore, the results did not support elaborative processing, an essential part of LOP theories [3-6], as an explanation for the effects of synopses at presentation on recognition. However, the hit and $A^{\prime}$ results did show encoding specificity for believable 


\section{Psychology \& Psychological Research International Journal}

headlines, but not for unbelievable headlines. One possible reason that encoding specificity could not account for the unbelievable headline results is that they produced extremely high recognition, which could not improve via matching synopsis conditions across presentation and test (i.e., encoding specificity). The possibility of such a ceiling effect was examined in Experiment 3 with a longer retention interval (7 days).

\section{Experiment 3}

Experiment 3 replicated the procedure in Experiment 2 with a 7-day retention interval. Although the exact same stimuli were used in this experiment as in Experiment 2, different participants took part in the two experiments. Based on the findings in Experiment 2 and a persistent encoding specificity effect for Ray and Reingold (2003) across different retention intervals (e.g., 1 and 2 days), we expected to find encoding specificity for the initially believable news headlines across the 7-day retention interval. However, if a ceiling effect for recognition explains the absence of encoding specificity for unbelievable headlines in Experiment 2, encoding specificity should be demonstrated for both the believable and unbelievable headlines across the 7-day retention interval in Experiment 3.

\section{Method}

\section{Participants}

A group of 34 undergraduate psychology students from Christopher Newport University participated in Experiment 3 to earn extra course credit. The participants included both male and female adults between 18 and 25 years of age. All participants were treated in accordance to with the "Ethical principles of psychologists and code of conduct" [9].

\section{Materials, Procedure, Measures, Design and Analysis}

The materials, procedure, measures, design, and analyses used in Experiment 2 were also used in Experiment 3. The false alarm rates were low in Experiment 3. The false alarm rates were low with a rate of .017 overall, and rates of $0, .034, .025$, and .010 for believable headlines without synopses, believable headlines with synopses, unbelievable headlines without synopses, and unbelievable headlines with synopses, respectively.

\section{Results}

Hits

The initially unbelievable headlines $(M=.806, S E=$ $.030)$ were recognized better than the initially believable headlines $(M=.657, S E=.044), F(1,33)=22.354, p<$ .001 , power $=.996, \eta^{2}=.404$. No other main effects were significant, nor were any of the 2-way interactions statistically significant. However, the Headline Type $\mathrm{x}$ Presence of Synopses at Presentation x Presence of Synopsis at Test interaction was statistically significant, $F(1,33)=6.703, p=.014$, power $=.710, \eta^{2}=.169$.

A breakdown of the 3-way interaction yielded a significant Presence of Synopses at Presentation $\mathrm{x}$ Presence of Synopses at Test interaction for initially believable headlines, $F(1,33)=5.528, p=.025$, power $=$ $.626, \eta^{2}=.143$, but not for initially unbelievable headlines, $F(1,33)=1.202, p=.281$, power $=.187, \eta^{2}=.035$. Further analysis of the 2-way interaction for believable headlines into paired samples $t$-tests yielded one significant result. Specifically, recognition was significantly higher for initially believable headlines with congruent conditions when synopses were present at presentation $\left(M_{\text {Synopses }}\right.$ present at presentation and test $=.706, S E=.058 ; M_{\text {Synopses present at }}$ presentation but absent at test $=.569, S E=.061), t(33)=2.291, p=$ .028 , power $=.604, \eta^{2}=.137$, and nominally higher when synopses were absent at presentation ( $M_{\text {Synopses absent at }}$ presentation but present at test $=.647, S E=.049 ; M_{\text {Synopses absent at }}$ presentation and test $=.706, S E=.061), t(33)=-0.924, p=.362$, power $=.146, \eta^{2}=.025$.

\section{$A^{\prime}$}

A' could not be calculated for 10 participants across all the conditions for the initially believable headlines (data for 7 participants for the initially believable headlines, 2 participants for the initially unbelievable headlines, and 1 participant for both headlines). Therefore, the data for these participants were dropped from the $\mathrm{A}^{\prime}$ analyses. The initially unbelievable headlines $(M=.959, S E=.007)$ were recognized better than the initially believable headlines $(M=.935, S E=.011), F(1,23)=8.782, p<.007$, power $=$ $.810, \eta^{2}=.276$. No other main effects were significant, but the Headline Type $\mathrm{x}$ Presence of Synopses at Test interaction was statistically significant, $F(1,23)=4.372, p$ $<.007$, power $=.517, \eta^{2}=.160$, which showed that the presence of synopses at test enhanced the recognition of the believable headlines, not the unbelievable headlines. The Headline Type $x$ Presence of Synopses at Presentation 
$\mathrm{x}$ Presence of Synopses at Test interaction was also statistically significant, $F(1,23)=6.980, p=.015$, power $=$ $.716, \eta^{2}=.233$ (see Figures $2 \mathrm{~A}$ and $2 \mathrm{~B}$ ).
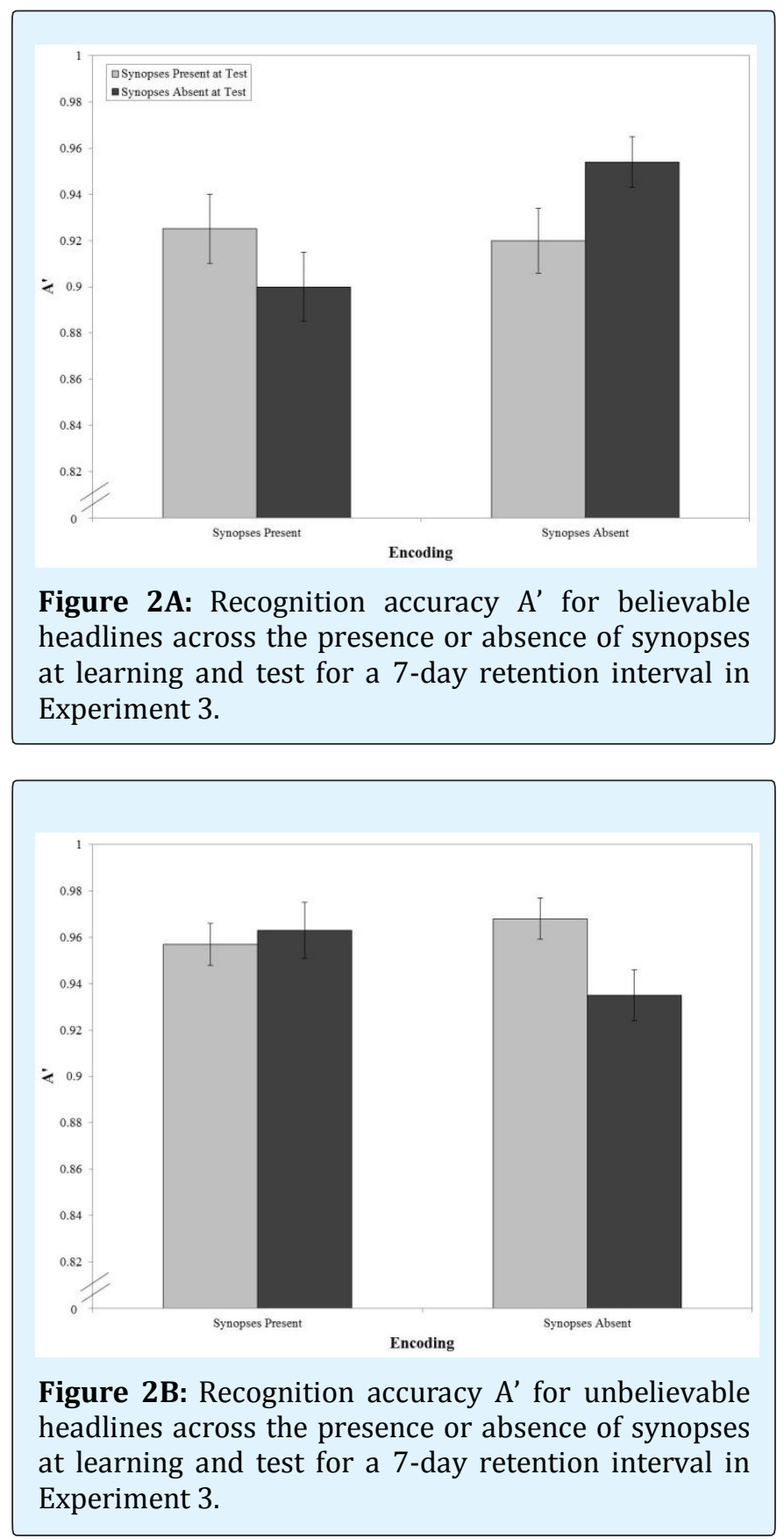

Further analysis of the 3-way interaction yielded a significant Presence of Synopses at Presentation $\mathrm{x}$ Presence of Synopses at Test interaction for initially believable headlines, $F(1,25)=9.416, p=.005, \phi=.839$, $\eta^{2}=.274$ (Figure 2A), but not for initially unbelievable headlines, $F(1,30)=1.506, p=.229$, power $=.221, \eta^{2}=$ .048 (Figure 2B). Additional analysis of the 2-way interaction for believable headlines into paired samples $t$ tests yielded one significant result. Specifically, recognition was nominally higher for initially believable headlines with congruent conditions when synopses were present at presentation $\left(M_{\text {Synopses present at presentation and test }}=\right.$ .937, $S E=.014 ; M_{\text {Synopses present at presentation but absent at test }}=.920$, $S E=.014), t(26)=1.392, p=.176$, power $=.268, \eta^{2}=.069$, and significantly higher when synopses were absent at presentation ( $M_{\text {Synopses absent at presentation but present at test }}=.899$, $\left.S E=.017 ; M_{\text {Synopses absent at presentation and test }}=.954, S E=.011\right)$, $t(28)=-3.011, p=.005$, power $=.828, \eta^{2}=.245$.

\section{Discussion}

Like Experiment 2, the presence of synopses at presentation did not enhance recognition, which did not show that reiterative synopses enhanced elaborative processing, as per the LOP framework [3]. Interestingly, the $A^{\prime}$ results showed that synopses at test enhanced recognition of believable headlines, which suggests that synopses at test may act as retrieval cues. Although the 3way and 2-way interactions for hits and $\mathrm{A}^{\prime}$ in Experiment 3 clearly demonstrated encoding specificity for believable headlines at a general level, further analysis of the 2-way interactions for believable headlines into individual mean comparisons only demonstrated partial encoding specificity. In fact, the locus of the partial encoding specificity effects for hits and $A^{\prime}$ were different, due to the relatively large difference in false alarms across believable foil headlines with and without synopses. Therefore, encoding specificity was demonstrated, but it was not as pervasive for the 7-day retention interval in Experiment 3 as it was for the 2-day retention interval in Experiment 2. Moreover, the results for the unbelievable headlines stood in stark contrast to the results for the believable headlines, as encoding specificity was not demonstrated for unbelievable headlines across the 7-day retention interval in Experiment 3. These results do not support the ceiling effect explanation posed as an alternative to account for the lack of encoding specificity for unbelievable headlines in Experiment 2.

One limitation with the methodology of Experiments 2 and 3 is that it did not rotate the headline groupings through the different synopsis conditions, even though they were balanced using pre-ratings from a different group of participants. Therefore, the results of these experiments could have been due to the grouping of the headlines rather than the conditions. Experiments 4 and 5 were created to rectify this problem by replicating 


\section{Psychology \& Psychological Research International Journal}

Experiments 2 and 3, respectively, and rotating the headline groupings across synopsis conditions.

\section{Experiment 4}

Experiment 4 replicated the methodology used in Experiment 2 with a 2-day retention interval, but we rotated the synopsis grouping conditions across groupings of headlines in a Latin square. Much like Experiment 2, the presence of synopses at presentation should enhance recognition if synopses enhance elaborate processing, as per the LOP framework [3]. In addition, synopses should only enhance recognition for matching conditions across presentation and test to support encoding specificity as an explanation for the effects of synopses on the recognition of news headlines.

\section{Method}

\section{Participants}

All 75 students were undergraduate psychology students from a small, public southeastern university who participated in the experiment to earn extra credit. The participants included both male and female adults between 18 and 25 years of age. All participants were treated in accordance to with the "Ethical principles of psychologists and code of conduct" [9].

\section{Materials}

The same two main questionnaires used in Experiments 2 and 3 were used in Experiment 4 as well, but these two main questionnaires were changed and paired to create four combinations that pertained to way synopsis groupings were combined with headline groupings. In contrast to Experiments 2 and 3, we did not want to pair a single synopsis grouping with a single grouping of headlines for all participants because this singular pairing confounds headline groupings with synopsis groupings. Instead, we created four headline groupings and rotated synopsis groupings across these headline groupings. Just as in Experiments 2 and 3, headline groupings were created based on believability ratings from prior studies to ensure that each headline group was as similar in average believability rating as possible across the believable or unbelievable headlines, respectively. Groups of believable and unbelievable headlines were paired to create a headline grouping, such as headline grouping $\mathrm{A}$.

We rotated the synopsis groupings across headline groupings using a Latin square to create four synopsis grouping orders that participants experienced in a repeated-measures manipulation. One such synopsis grouping order showed synopses at presentation and test for headlines in headline grouping $\mathrm{A}$, synopses at presentation but not test for headlines in headline grouping B, no synopses at presentation but synopses at test for headlines in headline grouping $\mathrm{C}$, and no synopses at presentation nor test for headlines in headline grouping D. The other synopsis grouping orders paired the synopsis groupings in the aforementioned order across headline groupings in the following orders: BDAC, CADB, and DCBA.

\section{Procedure}

Participants each received a questionnaire containing 24 headlines (12 believable headlines and 12 unbelievable headlines) at presentation. Participants read each headline and rated its believability on the scale that followed each headline. Once each participant was finished rating the believability of the headlines, the researcher collected the questionnaires. Two days later at test, the same participants returned and they were each given a 48-item questionnaire, which contained 24 believable headlines and 24 unbelievable headlines. Twelve of the 24 believable headlines were presented at both presentation and test, whereas 12 of the 24 unbelievable headlines were presented at both presentation and test. Participants then rated the believability of each headline as well as circled the headlines that they believed they recognized from the encoding questionnaires.

\section{Measures, Design, and Analyses}

Hits and A' (hits corrected for false alarms) served as the dependent variables in the experiment. Hits were calculated across eight headline categories. These categories included initially believable headlines with synopses present at both presentation and test, initially believable headlines with synopses present only at presentation, initially believable headlines with synopses present only at test, and initially believable headlines with no synopses present at either presentation or test. The headline categories also included initially unbelievable headlines with synopses at both presentation and test, initially unbelievable headlines with synopses present only at presentation, initially unbelievable headlines with synopses present only at test, and initially unbelievable headlines with no synopses present at either presentation or test.

False alarms were calculated across four categories, which included believable and unbelievable headlines with and without synopses at test. A' was computed as 
described in Experiment 1. The false alarm rates were low with a rate of .017 overall, and rates of $.014, .020, .022$, and .011 for believable headlines without synopses, believable headlines with synopses, unbelievable headlines without synopses, and unbelievable headlines with synopses, respectively. The hit and A' measures for a participant in a headline category were placed into a 2 (Headline Type) $x \quad 2$ (Presence of Synopsis at Presentation) x 2 (Presence of Synopsis at Test) $\mathrm{x} 4$ (Synopsis Grouping Order) mixed-factorial design in which synopsis grouping order was the only between groups factor. The data were analyzed using ANOVA. Paired-samples $t$-tests were conducted to understand interactions.

\section{Results}

The significant interactions involving grouping order were not predicted and were not analyzed further. However, the presence of these significant interactions indicate that any lower order effects involving factors other than grouping order in that interaction were qualified by and suspect as a result of it. The solution to reevaluating the reliability of the suspect effect(s) was to place the sum of squares for the significant interaction involving grouping order into the error term for the suspect effect and recalculate the $F$-value for it. This statistical solution is the same one used to calculate effects for random factors [27].

\section{Hits}

The initially unbelievable headlines $(M=.944, S E=$ .016) were better recognized than the initially believable headlines $(M=.895, S E=.016), F(1,71)=14.426, p<$ .001 , power $=.963, \eta^{2}=.169$. In addition, headlines were recognized better with synopses at test $(M=.933, S E=$ $.016)$ than without synopses at test $(M=.906, S E=.015)$, $F(1,71)=7.507, p=.008$, power $=.771, \eta^{2}=.096$. The Synopses at Presentation x Synopsis Grouping Order interaction was statistically significant, $F(1,71)=4.153, p$ $=.009$, power $=.833, \eta^{2}=.149$. Moreover, the Headline Type $x$ Presence of Synopses at Presentation $x$ Presence of Synopses at Test interaction was statistically significant, $F(1,71)=4.309, p=.042$, power $=.535, \eta^{2}=.057$.

Further analysis of the 3-way interaction yielded a statistically significant Presence of Synopses at Presentation x Presence of Synopses at Test interaction for initially believable headlines, $F(1,74)=4.998, p=$ .028 , power $=.597, \eta^{2}=.063$, but not for initially unbelievable headlines, $F(1,74)=0.225, p=.636$, power $=$ $.076, \eta^{2}=.003$. Additional analysis of the 2-way interaction for initially believable headlines showed that recognition was significantly higher for initially believable headlines with congruent conditions when synopses were present at presentation $\left(M_{\text {Synopses present at presentation and test }}=\right.$ $.938, S E=.020 ; M_{\text {Synopses present at presentation but absent at test }}=.849$, $S E=.028), t(74)=3.476, p=.001$, power $=.929, \eta^{2}=.140$, but only nominally higher when synopses were absent at presentation ( $M_{\text {Synopses absent at presentation but present at test }}=.889$, $\left.S E=.026 ; M_{\text {Synopses absent at presentation and test }}=.898, S E=.021\right)$, $t(74)=-0.276, p=.784$, power $=.059, \eta^{2}=.001$.

\section{$\mathbf{A}^{\prime}$}

$\mathrm{A}^{\prime}$ could not be calculated for 4 participants; the data for these participants were dropped from the overall $\mathrm{A}^{\prime}$ analyses. The initially unbelievable headlines $(M=.984$, $S E=.003)$ were better recognized than the initially believable headlines $(M=.973, S E=.003), F(1,69)=$ 8.372, $p=.005$, power $=.814, \eta^{2}=.108$. In addition, headlines were recognized better with synopses at test $(M$ $=.983, S E=.003)$ than without synopses at test $(M=.974$, $S E=.003), F(1,69)=7.814, p=.007$, power $=.787, \eta^{2}=$ .102. The Synopses at Presentation $x$ Synopsis Grouping Order interaction was statistically significant, $F(1,69)=$ $3.290, p=.026$, power $=.728, \eta^{2}=.125$. Moreover, the Headline Type $\mathrm{x}$ Presence of Synopses at Presentation $\mathrm{x}$ Presence of Synopses at Test interaction was statistically significant, $F(1,69)=5.248, p=.025$, power $=.618, \eta^{2}=$ .071 (see Figures $3 \mathrm{~A}$ and $3 \mathrm{~B}$ ).

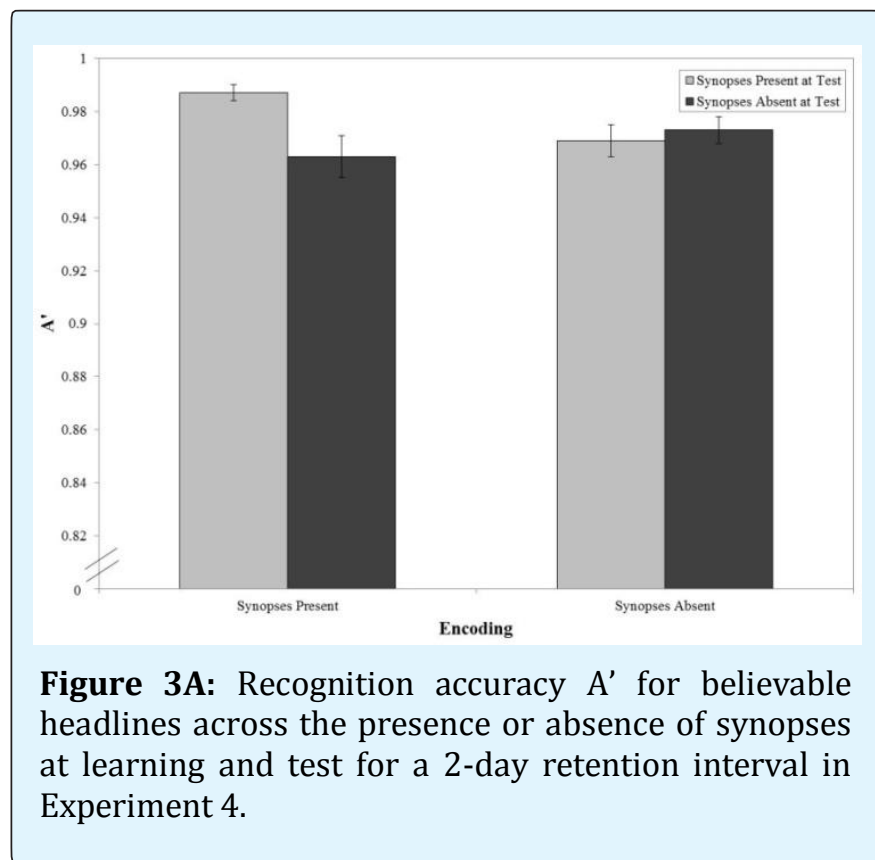




\section{Psychology \& Psychological Research International Journal}

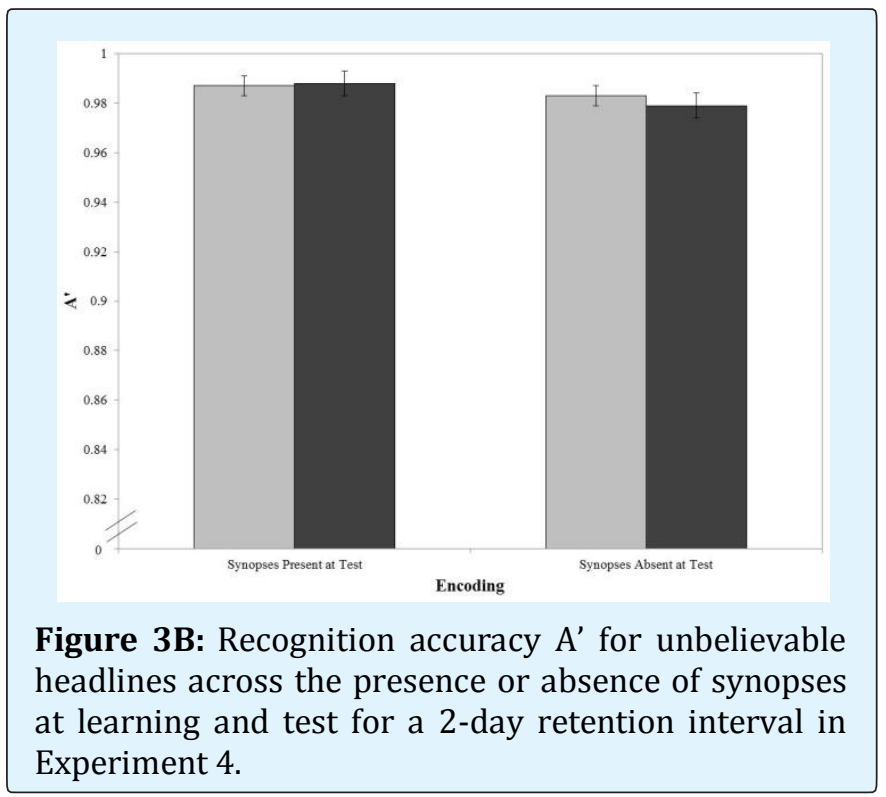

Further analysis of the 3-way interaction yielded a statistically significant Presence of Synopses at Presentation x Presence of Synopses at Test interaction for initially believable headlines, $F(1,69)=5.022, p=$ .028 , power $=.599, \eta^{2}=.068$ (Figure 3A), but not for initially unbelievable headlines, $F(1,70)=0.197, p=.659$, power $=.072, \eta^{2}=.003$ (Figure 3B). Additional analysis of the 2-way interaction into paired samples $t$-tests for initially believable headlines revealed that recognition was significantly higher for initially believable headlines with congruent conditions when synopses were present at presentation ( $M_{\text {Synopses present at presentation and test }}=.986, S E=$ .003; $M_{\text {Synopses present at presentation but absent at test }}=.963, S E=.008$ ), $t(72)=2.982, p=.004$, power $=.837, \eta^{2}=.110$, but only nominally higher when synopses were absent at presentation (M $M_{\text {Synopses absent at presentation but present at test }}=.969$, $\left.S E=.006 ; M_{\text {Synopses absent at presentation and test }}=.973, S E=.005\right)$, $t(73)=-0.493, p=.624$, power $=.077, \eta^{2}=.003$.

\section{Discussion}

The presence of synopses at presentation did not enhance recognition. Therefore, the results did not support elaborative processing, and the LOP framework $[3,6]$, as an explanation for the effects of synopses at presentation on recognition. However, the presence of synopses at test did enhance recognition, which suggests that synopses acted as a retrieval mechanism for believable and unbelievable headlines. Moreover, the results did provide some support for encoding specificity. Specifically, the results supported an encoding specificity explanation for believable headlines at a general level of analysis, but they only partially supported encoding specificity for believable headlines at a mean-level of comparison. Conversely, support for encoding specificity was not found for unbelievable headlines in Experiment 4. These results very closely matched the results from Experiment 3. As we stated in the discussion of Experiment 2, one reason that reiterative synopses may not have produced encoding specificity for unbelievable headlines is that the headlines produced extremely high recognition on their own, which could not be improved via matching synopsis conditions across presentation and test (i.e., encoding specificity). The possibility of such a ceiling effect was examined in Experiment 5 with a longer retention interval (7 days) and the thorough methodology of rotating synopsis conditions through headline groups used in Experiment 4.

\section{Experiment 5}

Experiment 5 replicated the same 7-day retention interval used in Experiment 3, but it rotated the synopsis conditions across groupings of headlines in a Latin square, as was done in Experiment 4. As stated in Experiments 1-4, the presence of synopses at presentation should enhance recognition if synopses enhance elaborate processing, as per the LOP framework [3]. In addition, synopses should only enhance recognition for matching conditions across learning and test to support encoding specificity as an explanation for the effects of synopses on recognition.

\section{Method}

\section{Participants}

A group of 34 undergraduate psychology students from Christopher Newport University participated in Experiments 3 to earn extra course credit. The participants included both male and female adults between 18 and 25 years of age. All participants were treated in accordance to with the "Ethical principles of psychologists and code of conduct" [9].

\section{Materials, Procedure, Measures, Design and Analysis}

The materials, procedure, measures, design, and analyses used in Experiment 4 were also used in Experiment 5 with the exception of using the 7-day retention interval that was used in Experiment 3. The false alarm rates were low in Experiment 5. Specifically, the overall false alarm rate was .023, and the false alarm rates were $.029, .015, .031$, and .018 for believable headlines without synopses, believable headlines with 
synopses, unbelievable headlines without synopses, and unbelievable headlines with synopses, respectively.

\section{Results}

The alpha level was set at .05. We further analyzed predicted interactions in Experiment 5.

\section{Hits}

The initially unbelievable headlines $(M=.959, S E=$ .007) were recognized better than the initially believable headlines $(M=.935, S E=.011), F(1,72)=20.094, p<$ .001 , power $=.810, \eta^{2}=.993$. No other main effects were significant, but three 2-way interactions were significant. Specifically, the Synopses at Presentation $\mathrm{x}$ Synopsis Grouping Order interaction was statistically significant, $F(1,72)=10.030, p<.001$, power $=.997, \eta^{2}=.295$, and the Synopses at Test $x$ Synopsis Grouping Order interaction was statistically significant, $F(1,72)=3.282, p$ $=.026$, power $=.728, \eta^{2}=.120$. Importantly, the Presence of Synopsis at Presentation x Presence of Synopsis at Test interaction was also statistically significant, $F(1,72)=$ 12.039, $p=.001$, power $=.928, \eta^{2}=.143$ (see Figure 4a). This 2-way interaction was qualified by a significant Presence of Synopsis at Presentation $x$ Presence of Synopsis at Test $\mathrm{x}$ Synopsis Grouping Order interaction, $F(1,72)=3.600, p=.017$, power $=.771, \eta^{2}=.130$.

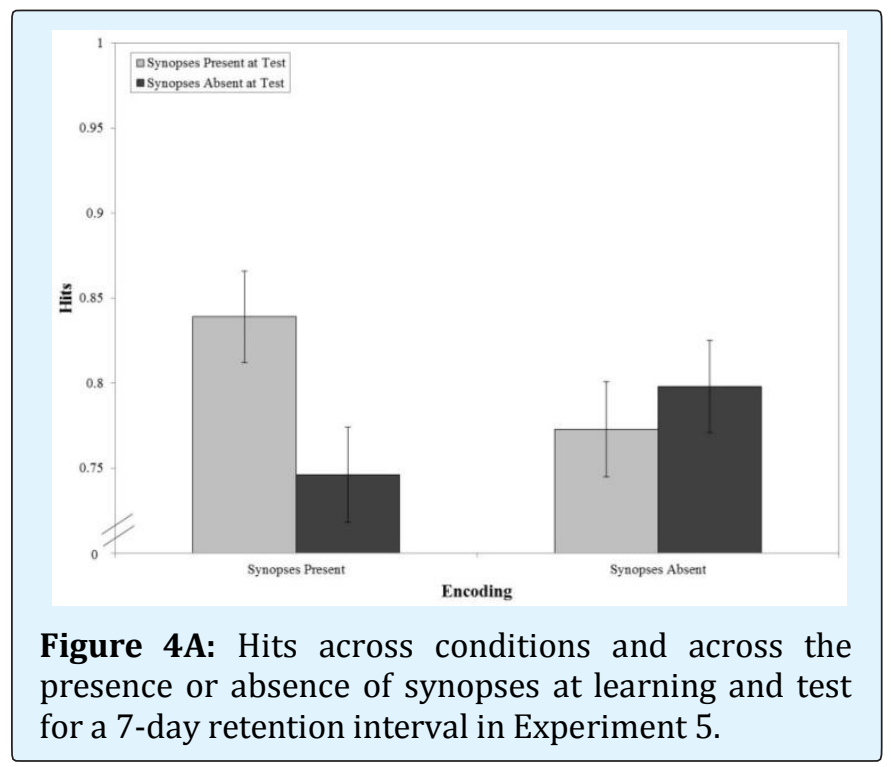

In order to statistically control for the 3-way interaction, we used the sum of squares and the degrees of freedom for this interaction and we combined it with the error term for the Presence of Synopses at Presentation x Presence of Synopses at Test to create a pooled error term, and we recalculated the $F$-value. The resulting Presence of Synopses at Presentation x Presence of Synopses at Test interaction was still statistically significant, $F(1,75)=10.912, p<.01$. Recognition was higher for headlines with congruent conditions when synopses were present at presentation ( $M_{\text {Synopses present at }}$ presentation and test $=.839, S E=.027 ; M_{\text {Synopses present at presentation but }}$ absent at test $=.746, S E=.028), t(75)=3.846, p=.024$, power $=.967, \eta^{2}=.165$. Recognition was also marginally higher for headlines with congruent conditions when synopses were absent at presentation ( $M_{\text {Synopses absent at presentation but }}$ present at test $=.773, S E=.028 ; M_{\text {Synopses absent at presentation and test }}=$ $.798, S E=.027), t(75)=-1.030, p=.306$, power $=.174, \eta^{2}$ $=.014$.

\section{$\mathbf{A}^{\prime}$}

$A^{\prime}$ could not be calculated for 15 participants across all the conditions for the initially believable headlines. Therefore, the data for these participants were dropped from the $A^{\prime}$ analyses. The initially unbelievable headlines $(M=.966, S E=.005)$ were recognized better than the initially believable headlines $(M=.948, S E=.005), F(1$, $57)=8.817, p=.004$, power $=.831, \eta^{2}=.134$. In addition, headlines were recognized better with synopses at test $(M$ $=.963, S E=.004)$ than without synopses at test $(M=.951$, $S E=.006), F(1,57)=4.762, p=.033$, power $=.574, \eta^{2}=$ .077. The Synopses at Presentation x Synopsis Grouping Order interaction was statistically significant, $F(1,57)=$ 4.499, $p=.007$, power $=.191, \eta^{2}=.859$. Importantly, the Presence of Synopses at Presentation $x$ Presence of Synopses at Test interaction was also statistically significant, $F(1,57)=5.681, p=.021$, power $=.649, \eta^{2}=$ .091 (see Figure 4b). This 2-way interaction was qualified by a significant Presence of Synopses at Presentation $\mathrm{x}$ Presence of Synopses at Test $x$ Synopses Grouping Order interaction, $F(1,57)=6.039, p=.001$, power $=.947, \eta^{2}=$ .241 .

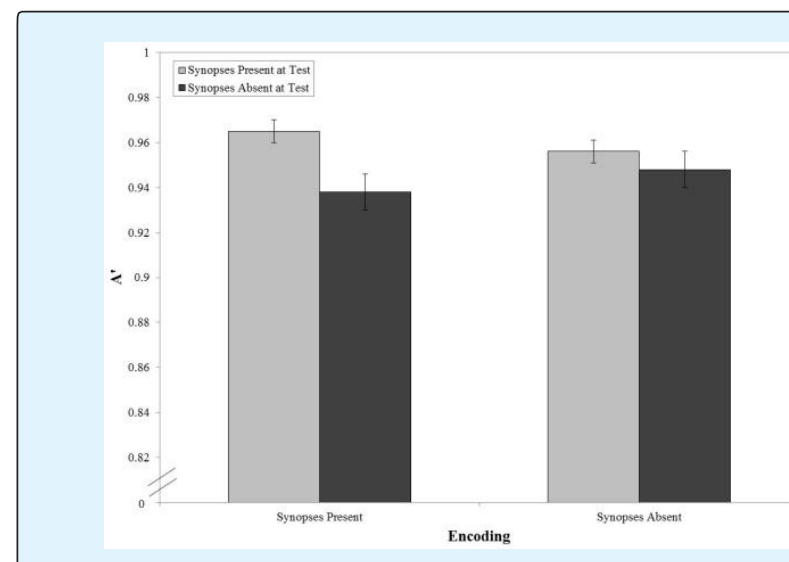

Figure 4B: Recognition accuracy A' across conditions across the presence or absence of synopses at learning and test for a 7-day retention interval in Experiment 5. 


\section{Psychology \& Psychological Research International Journal}

In order to statistically control for the 3-way interaction, we placed sum of squares and the degrees of freedom for this interaction into the error term for the Presence of Synopses at Presentation x Presence of Synopses at Test to create a pooled error term, and we recalculated the $F$-value. The resulting Presence of Synopsis at Presentation $x$ Presence of Synopsis at Test interaction was still statistically significant, $F(1,60)=$ 4.615, $p<.01$. Follow-up analyses revealed that recognition was significantly higher for headlines with congruent conditions when synopses were present at presentation $\left(M_{\text {Synopses present at presentation and test }}=.968, S E=\right.$ $.005 ; M_{\text {Synopsis present at presentation but absent at test }}=.938, S E=.008$ ), $t(65)=3.481, p=.001$, power $=.929, \eta^{2}=.157$, but not when synopses were absent at presentation $\left(M_{\text {Synopsis absent }}\right.$ at presentation but present at test $=.956, S E=.005 ; M_{\text {Synopsis absent at }}$ presentation and test $=.954, S E=.006), t(64)=0.337, p=.737$, power $=.113, \eta^{2}=.002$.

\section{Discussion}

Like Experiments 2-4, the presence of synopses at presentation did not enhance recognition, which did not support elaborative processing, as per the LOP framework [3]. Much like Experiment 4, however, synopses at test enhanced recognition for the A' measure, which suggests that reiterative synopses acted as a retrieval mechanism for the recognition of news headlines. More importantly, encoding specificity was shown for both believable and unbelievable headlines at a general level through the significant Presence of Synopses at Presentation by Presence of Synopses at Test interaction. Through the mean level comparisons further breaking down and examining the interaction, encoding specificity was demonstrated for the hit and A' measures when synopses were present at presentation, but not when synopses were absent at presentation. As both hit and $A^{\prime}$ measures showed a pattern of results consistent with encoding specificity for believable and unbelievable headlines and both measures showed significant 2-way interactions consistent with encoding specificity, the results partially demonstrate encoding specificity.

The partial encoding specificity for unbelievable headlines also partially supports the ceiling effect explanation proposed in the discussions of Experiments 2 and 4. In particular, unbelievable headlines produce extremely high recognition, which cannot be improved by matching synopsis conditions (i.e., encoding specificity). However, the longer retention interval used in Experiment 5 lowered recognition and allowed matching synopsis conditions (when synopses were present at presentation) to enhance recognition. The fact that this outcome was produced in Experiment 5 and not Experiment 3 may be explained by the different methodologies used in the two experiments. Specifically, the confounding of headline groupings with synopsis conditions in Experiment 3 could have hidden the encoding specificity effects for unbelievable headlines, whereas the cleaner methodology in Experiment 5, rotating headline groupings across synopsis conditions, allowed those effects to be produced when synopses were present at presentation.

\section{General Discussion}

None of the results from the five experiments show that synopses at presentation enhance recognition. Therefore, the recognition results for synopses cannot be accounted for by the LOP framework [3]. However, the presence of synopses at test in Experiments 3 through 5 did enhance recognition, which suggests that synopses acted as a retrieval mechanism across headline conditions. Moreover, the recognition results for believable headlines consistently showed encoding specificity at a general level of analysis in the form of significant 2- and 3-way interactions. However, the meanlevel comparisons that further analyzed the significant interactions showed, in all cases except for the $\mathrm{A}^{\prime}$ analyses in Experiment 3, encoding specificity when synopses were present at presentation.

Encoding specificity was demonstrated for unbelievable headlines in Experiment 5. The experiment used a long retention interval to reduce recognition, which allowed matching synopses conditions to improve recognition of unbelievable headlines, but only when synopses were present at presentation. The matching synopsis conditions in Experiments 2 and 4 likely did not enhance recognition for unbelievable headlines because recognition was high for the 2-day retention interval and could not increase. The reason that matching synopses conditions aided recognition in Experiment 5 but not in Experiment 3, even though both experiments used a 7 day retention interval, pertains to the different methodologies used in the two experiments. As Experiment 5 used the cleaner procedure, the results from that experiment should be interpreted with more weight than the results from Experiment 3. Therefore, encoding specificity was reliably demonstrated for believable and unbelievable news headlines via matching and mismatching synopsis conditions when reiterative synopses were present at presentation.

The results of the current study provide some support for Tulving and Thomson's (1973) claim that encoding 


\section{Psychology \& Psychological Research International Journal}

specificity pertains to "all known phenomena of episodic memory and retrieval" [13]. The results of the current study replicate a large body of work on encoding specificity showing superior retrieval for matching conditions across presentation and test across a variety of manipulations [13,18,20-22,25-29]. These results also extend the results of Ray and Reingold (2003) who demonstrated persistent encoding specificity across different retention intervals as well [29].

Although the results in the current study extend past work demonstrating encoding specificity, the encoding specificity shown in the current study for synopses and media headlines was limited to presentation. Apparently, the removal of synopses at test that were present at presentation hinders recognition, whereas the addition of synopses at test that were absent at presentation does not affect recognition. In other words, subtraction of stimulus details, such as synopses, is more influential than addition of those stimulus details in the context of news headlines. Future research should determine whether the "subtraction is more influential than addition" principle demonstrated for synopses, news headlines, and recognition in the current study generalizes to other media stimuli.

The findings of the current study provide newspaper editors with at least two methods to enhance the degree that their readers remember their headlines: 1) present unbelievable news headlines, and 2) do not remove information, especially reiterative information, from news headlines across the initial presentation and further presentations. Unbelievable news headlines should be remembered better than believable news headlines because they grab consumers' attention due to their sensational nature [2]. In addition, the consistency of the information contained in believable news headlines should ensure that they are remembered across time. Based on the findings in the current study, this same principle should extend to unbelievable news headlines as long as the initial presentation of the information and future presentations are separated by lengthy intervals (i.e., 7 days).

Despite the strengths of the present research, one criticism is that memory was tested using recognition rather than recall. This fact is not a concern for several reasons. First, the notion that encoding specificity is limited to recall measures alone does not support Tulving and Thomson's (1973) strong claim about encoding specificity generalizing to all retrieval phenomena. Second, recognition has been used in past studies demonstrating encoding specificity [22-24]. Third, encoding specificity was demonstrated for believable and unbelievable headlines when synopses were present at presentation in the current study. Therefore, recognition is a viable measure of retrieval for procedures examining encoding specificity via matching and mismatching stimulus conditions across presentation/encoding and test $[30,32]$.

In summary, the current study examined the effect of synopses at presentation/encoding and test on recognition for believable and unbelievable headlines. Unbelievable headlines were recognized better than believable headlines, replicating the work of Gibbons, et al. (2005) [1]. For both believable and unbelievable headlines, elaborative synopses at presentation decreased recognition, reiterative synopses at presentation did not affect recognition, and reiterative synopses at test enhanced recognition. These results show that synopses do not enhance encoding as one would expect based on LOP models, but they can act as a retrieval mechanism $[33,37]$. More importantly, matching reiterative synopsis conditions at presentation and test reliably enhanced recognition for believable and unbelievable headlines when synopses were present at presentation. These results extend encoding specificity to believable and unbelievable news headlines. Future research should replicate and extend the experiments in the current study to further target and examine the cognitive processes and conditions responsible for the way news headlines are remembered [38].

\section{References}

1. Gibbons JA, Lukowski AF, Walker RW (2005) Exposure increases the believability of unbelievable news headlines via elaborate cognitive processing. Media Psychology 7(3): 273-300.

2. Grabe ME, Zhou S, Lang A, Bolls PD (2000) Packaging television news: The effects of tabloid on information processing and evaluative responses. Journal of Broadcasting and Electronic Media 44(4): 581-598.

3. Craik FIM, Kester JD (2000) Divided attention and memory: Impairment of processing or consolidation? In: Tulving E (Ed.), Memory, consciousness, and the brain: The Tallinn Conference. Philadephia, PA: Psychology Press/Taylor \& Francis, 38-51.

4. Craik FI, Lockhart RS (1972) Levels of processing: A framework for memory research. Journal of Verbal Learning \& Verbal Behavior 11(6): 671-684. 


\section{Psychology \& Psychological Research International Journal}

5. Lockhart RS, Craik FI (1990) Levels of processing: A retrospective commentary on a framework for memory research. Canadian Journal of Psychology 44(1): 87-112.

6. Roediger HL, Gallo DA, Geraci L (2002) Processing approaches to cognition: The impetus from the levels of processing framework. Memory 10(5-6): 319-332.

7. Lang A (2000) The limited capacity model of mediated message processing. Journal of Communication 50(1): 46-70.

8. Shapiro MA, Lang A (1991) Making television reality: Unconscious processes in the construction of social reality. Communication Research 18(5): 685-705.

9. American Psychological Association (2010) 2010 Amendments to the 2002 "Ethical principles of psychologists and code of conduct". American Psychologist 65(5): 493.

10. Snodgrass JG, Corwin J (1988) Pragmatics of measuring recognition memory: Applications to dementia and amnesia. Journal of Experimental Psychology: General 117(1): 34-50.

11. Mulligan NW (1998) The role of attention during encoding in implicit versus explicit memory. Journal of Experimental Psychology 24(1): 27-47.

12. Thompson CP, Gibbons JA, Vogl RJ, Walker WR (1996) Autobiographical memory: Individual differences in using episodic and schematic information. In Payne DG, Conrad FG (Eds.). A synthesis of basic and applied approaches to human memory. New York: Lawrence Erlbaum Associates, 193-213.

13. Thomson DM, Tulving E (1970) Associative encoding and retrieval: Weak and strong cues. Journal of Experimental psychology 86(2): 255-262.

14. Tulving E (1983) Elements of episodic memory. New York: Oxford University Press.

15. Tulving E, Thomson DM (1973) Encoding specificity and retrieval processes in episodic memory. Psychological Review 80(3): 352-373.

16. Godden DR, Baddeley AD (1975) Context-dependent memory in two natural environments: On land and under water. British Journal of Psychology 66(3): 325-331.
17. Hannon B, Craik FIM (2001) Encoding specificity revisited: The role of semantics. Canadian Journal of Experimental Psychology 55(3): 231-243.

18. Smith SM, Glenberg A, Bjork RA (1978) Environmental context and human memory. Memory and Cognition 6(4): 342-353.

19. Weir W, May RB (1988) Environmental context and student performance. Canadian Journal of Education 13(4): 505-510.

20. Ley R, Huba M (1980) Encoding specificity and experimenter-supplied semantic associates as cues in recall. The Journal of General Psychology 102(1): 2731.

21. Liu CH, Ward J, Young AW (2006) Transfer between two- and three-dimensional representations of faces. Visual Cognition 13(1): 51-64.

22. Newman SE (1982) Some tests of the encoding specificity and semantic integration hypotheses. American Journal of Psychology 95(1): 103-123.

23. Newman SE, Frith U (1977) Encoding specificity vs. associative continuity. The Bulletin of the Psychonomic Society 10(1): 73-75.

24. Reder LM, Anderson JR, Bjork RA (1974) A semantic interpretation of encoding specificity. Journal of Experimental Psychology 102(4): 648-656.

25. Friestad M, Thorson E (1993) Remembering ads: The effects of encoding strategies, retrieval cues, and emotional response. Journal of Consumer Psychology 2(1): 1-23.

26. Leshner G, Coyle JR (2000) Memory for television news: Match and mismatch between processing and testing. Journal of Broadcasting and Electronic Media 44(4): 599-613.

27. Keppel G, Wickens TD (2004) Design and analysis: A researcher's handbook. $4^{\text {th }}$ Ed. New Jersey: PearsonPrentice Hall.

28. Spence I, Wong P, Rusan M, Rastegar N (2006) How color enhances visual memory for natural scenes. Psychological Science 17(1): 1-6.

29. Ray CA, Reingold EM (2003) Long-term perceptual specificity effects in recognition memory: The transformed pictures paradigm. Canadian Journal of Experimental Psychology 57(2): 131-137. 
30. Cacioppo JT, Petty RE, Kao CF, Rodriguez R (1986) Central and peripheral routes to persuasion: An individual difference perspective. Journal of Personality and Social Psychology 51(5): 1032-1043.

31. Engle RW, Tuholski SW, Laughlin JE, Conway ARA (1999) Working memory, short-term memory, and general fluid intelligence: A latent variable approach. Journal of Experimental Psychology General 128(3): 309-331.

32. Kane MJ, Engle RW (2000) Memory capacity, proactive interference, and divided attention: Limits on long-term memory retrieval. Journal of Experimental Psychology: Learning, Memory, and Cognition 26(2): 336-358.

33. Lanska M, Olds JM, Westerman DL (2013) Fluency effects in recognition memory. Are perceptual fluency and conceptual fluency interchangeable? Journal of Experimental Psychology: Learning, Memory, and Cognition 40(1): 1-11.
34. Macmillan NA, Creelman CD (1991) Detection theory: A user's guide. Cambridge: Cambridge University Press.

35. Petty RE, Cacioppo JT (1984) The effects of involvement on responses to argument quantity and quality: Central and peripheral routes to persuasion. Journal of Personality and Social Psychology 46(1): 69-81.

36. Rosen VM, Engle RW (1997) The role of working memory capacity in retrieval. Journal of Experimental Psychology: General 126(3): 211-227.

37. Whittlesea BWA (1993) Illusions of familiarity. Journal of Experimental Psychology: Learning, Memory, and Cognition 19(6): 1235-1253.

38. Whittlesea BWA, Jacoby LL, Girard K (1990) Illusions of immediate memory: Evidence of attributional basis for feelings of familiarity and perceptual quality. Journal of Memory and Language, 29: 716-732.

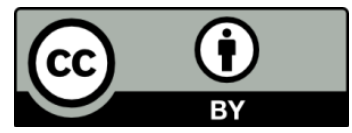

